\title{
Clinical Study \\ Serum Amyloid A Level in Egyptian Children with Familial Mediterranean Fever
}

\author{
Hala M. Lofty, ${ }^{1}$ Huda Marzouk, ${ }^{1}$ Yomna Farag, ${ }^{1}$ Mohammad Nabih,, ${ }^{1}$ Iman A. S. Khalifa, \\ Noha Mostafa, ${ }^{1}$ Ahmed Salah, ${ }^{1}$ Laila Rashed, ${ }^{2}$ and Kamal El Garf ${ }^{3}$ \\ ${ }^{1}$ Department of Pediatrics, Faculty of Medicine, Cairo University, Cairo, Egypt \\ ${ }^{2}$ Department of Medical Biochemistry, Faculty of Medicine, Cairo University, Cairo, Egypt \\ ${ }^{3}$ Department of Rheumatology, Faculty of Medicine, Cairo University, Cairo, Egypt
}

Correspondence should be addressed to Iman A. S. Khalifa; iman.khalifa@hotmail.com

Received 2 August 2016; Revised 19 November 2016; Accepted 22 November 2016

Academic Editor: Bruce M. Rothschild

Copyright (c) 2016 Hala M. Lofty et al. This is an open access article distributed under the Creative Commons Attribution License, which permits unrestricted use, distribution, and reproduction in any medium, provided the original work is properly cited.

Background and Objectives. SAA is an acute-phase reactant detected during an FMF attack or other inflammatory conditions. High SAA levels may increase the risk of amyloidosis. The aim of the study is to measure the serum amyloid A (SAA) level in a group of Egyptian children with familial Mediterranean fever (FMF) and study its various correlates, if any. Methods. The study enrolled seventy-one children with FMF. Results. SAA level was high in $78.9 \%$ of the studied patients with a mean of $81.62 \pm 31.6 \mathrm{mg} / \mathrm{L}$, and CRP was positive in $31 \%$ of patients. There was no significant releation between SAA level and any demographic or clinical manifestation. High SAA was more frequent in V726A allele (16.9\%) followed by M694V allele (12.3\%). Elevated SAA levels were more frequent in patients on low colchicine doses. Forty-five percent (45\%) of patients have low adherence to colchicine therapy. Interpretation and Conclusion. High SAA levels were detected two weeks after last FMF attack in a large percentage of Egyptian FMF children. This indicates that subclinical inflammation continues during attack-free periods, and SAA could be used as a marker of it.

\section{Introduction}

Familial Mediterranean fever (FMF) is the most common inherited autoinflammatory syndrome, with an autosomalrecessive pattern of inheritance [1]. The highest prevalence is among Turks, Arabs, Jews, and Armenian populations [2]. It is caused by Mediterranean fever (MEFV) gene mutation mapped onto the short arm of chromosome $16[3,4]$.

FMF is characterized by recurrent self-limited episodes of fever, peritonitis, arthritis, pleuritis, and erysipelas-like erythema $[5,6]$. The clinical attacks are accompanied by elevation of erythrocyte sedimentation rate (ESR) and acutephase reactants as C-reactive protein (CRP), serum amyloid A (SAA), and fibrinogen. These entire laboratory parameters usually return to normal in the attack-free periods [7]. However, it has been reported that subclinical inflammation may continue during the attack-free periods in some patients and may lead to the development of amyloidosis $[8,9]$ that maybe complicated by end-stage renal disease [10].
Amyloidosis is not necessarily related to the severity of the disease, as it may occur in patients with mild and infrequent attacks, as well as in asymptomatic patients who do not have any clinical manifestation of FMF (phenotype II). However, high levels of SAA, which is the precursor of the main protein related to the amyloid deposit in FMF, for prolonged duration are mandatory for the development of amyloidosis $[11,12]$.

Colchicine, the main line of treatment in FMF, is effective in preventing attacks in $60-70 \%$ of patients. However, $20-$ $30 \%$ of patients respond only partially to maximal tolerated doses of colchicine and $5 \%$ of patients are nonresponders. The dose of colchicine in children ranges between 1 and $2 \mathrm{mg} / \mathrm{day}$, guided by response and tolerability $[13,14]$. However, SAA may be elevated in some patients in attack-free periods. In such patients, an increase in colchicine dose was reported to result in further decrease in SAA level $[12,15]$, thereby limiting the development of amyloidosis. Some studies reported poor compliance to colchicine in $40 \%$ of patients, which may 
be a contributing factor to colchicine nonresponse, requiring more efforts to educate patients and their caregivers and to solve any problems leading to this incompliance [16].

It was, therefore, intriguing to measure the SAA levels in Egyptian FMF children two weeks following the last attack and study their various correlates and value in detecting subclinical inflammation and rates of incompliance and plan for management.

\section{Material and Methods}

The study included 71 children with FMF, 36 males and 35 females. Patients were diagnosed according to the new pediatric FMF criteria $[6,17]$, which requires the presence of two or more of the following five criteria: fever, abdominal pain, chest pain, arthritis, and family history of FMF. Patients were followed up at the Pediatric Rheumatology Clinic of the Cairo University Specialized Pediatric Hospital during the period of January 2014 to May 2015.

Inclusion criteria for the study included age of disease onset before 18 years and all patients should have had an attack at least two weeks prior to the time of serum amyloid measurement.

The Cairo University Clinical Research Ethics Committee approved the study protocol, and informed consents were obtained from parents of all patients.

Patients were subjected to detailed history taking, including age at onset of symptoms, age at diagnosis, disease duration, features of attacks (frequency, duration, and clinical manifestations), and dose of colchicine taken by patients. Patients were divided into 2 groups according to the current dose of colchicine: patients on low dose " $<1.5 \mathrm{mg} /$ day" and the others on high dose " $\geq 1.5 \mathrm{mg} /$ day". For all patients duration of colchicine treatment and adherence to colchicine (adherence being defined as no colchicine dose missed in at least the last 6 months before the time of study) were recorded. Colchicine unresponsiveness defined clinically as the occurrence of at least one attack per month despite daily treatment with $2 \mathrm{mg}$ of colchicine or more was also recorded $[7,8]$.

Patients were subjected to thorough physical examination, and the following laboratory tests were performed: complete blood count (CBC, using blood cell counter, CellDyn 3700; Abbott), CRP (screened by latex agglutination test [18] and confirmed by nephelometry analysis), ESR [19], and urine analysis, besides assessment of serum amyloid A (SAA) level. The results of MEFV gene mutations testing were recorded from the patients' files.

For determination of SAA level, blood samples were withdrawn from patients, left to clot, and then centrifuged at $10000 \mathrm{rpm}$ for 20 minutes. The separated serum was aspirated and stored frozen at $-80^{\circ} \mathrm{C}$ until analysis of SAA, which was done by ELISA kits supplied by Cusabio, USA, according to manufacturer's instruction [20]. Each sample was tested in duplicate and the average was taken. A cutoff level of $30 \mathrm{mg} / \mathrm{L}$ SAA level was chosen to reflect a real increase in its level.

Measurement of SAA is usually done in our patients to evaluate disease activity and response to colchicine and to evaluate risk of amyloidosis in patients with frequent attacks despite maximum tolerated dose of colchicine.
TABLE 1: Clinical characteristics of the studied FMF patients during the FMF attack.

\begin{tabular}{lc}
\hline Variables & Frequency \\
\hline Fever & $64(90.1 \%)$ \\
Abdominal pain & $70(98.6 \%)$ \\
Chest pain & $49(69 \%)$ \\
Myalgia & $28(39.4 \%)$ \\
Arthritis & $25(35.2 \%)$ \\
Arthralgia & $53(74.6 \%)$ \\
Rash & $9(12.7 \%)$ \\
Oral ulcers & $17(23.9 \%)$ \\
Testicular affection & $4(11.1 \%)(4 / 36$ males $)$ \\
\hline
\end{tabular}

2.1. Statistical Analysis. Data analysis was performed through Statistical Package of Social Sciences (SPSS) software program for windows version 21. Data were expressed as mean and standard deviation for quantitative variables and number and percentage for qualitative ones. Comparison was performed through Chi-square or Fisher's exact test for qualitative variables and independent sample $t$-test for quantitative ones. Pearson correlation coefficient was calculated to signify the association of quantitative variables. $P$ values $<0.05$ were considered significant.

\section{Results}

The study included 71 children with FMF, 36 males and 35 females, with a mean age of $9.0 \pm 3.7$ years. The mean age at disease onset was $5.0 \pm 2.9$ years, and mean age at disease diagnosis was $7.1 \pm 3.4$ years. The mean disease duration was $4.0 \pm 2.8$ years. Six patients $(8.5 \%)$ had family history of FMF, 6 patients $(8.5 \%)$ had family history of renal disease, and 25 patients $(35.2 \%)$ had consanguineous parents. Ten patients (14.1\%) had proteinuria.

With regard to the distribution of MEFV gene mutation among the studied patients, 46 patients $(64.8 \%)$ were heterozygous, 13 patients $(18.3 \%)$ were compound heterozygous, and 12 patients (16.9\%) were homozygous. The allelic frequency of MEFV mutations was V726A (32.4\%), M694V (23.9\%), M694I (23.9\%), E148Q (22.5\%), and M680I (15.5\%).

All patients were on colchicine therapy, with mean duration of $1.9 \pm 1.8$ years. Forty-five percent $(45 \%)$ of patients have low adherence to colchicine therapy, mainly due to diarrhea or social and economic factors, $16 \%$ medium adherence and 39\% high adherence.

The frequency of attacks in the studied patients was $2.9 \pm$ $2.8 /$ month, with a mean duration of $1.6 \pm 1.6$ days. The clinical characteristics of FMF patients during attacks are presented in Table 1.

The results of laboratory investigations at the time of study are displayed in Table 2 . High SAA levels $(\geq 30 \mathrm{mg} / \mathrm{L})$ were found in $78.9 \%$ of patients with a mean \pm SD of $81.62 \pm 31.6 \mathrm{mg} / \mathrm{L}$. Further, $70.4 \%$ of the studied patients had increased ESR, with a mean \pm SD of $38.1 \pm 16.7 \mathrm{~mm} / \mathrm{hr}$, and only $31 \%$ had high CRP level, with a mean value \pm SD of $15.4 \pm 12.8$. However, no significant correlation was found 
TABLE 2: Laboratory findings of studied FMF patients at the time of study.

\begin{tabular}{lcc}
\hline Variable & Range & Mean \pm SD \\
\hline Hemoglobin $(\mathrm{gm} / \mathrm{dL})$ & $10.1-13.9(71)$ & $12.3 \pm 0.9$ \\
Total leucocytic count $(\times 1000 / \mu \mathrm{L})$ & $3.8-23.4(71)$ & $7.7 \pm 3.2$ \\
Platelet count $(\times 1000 / \mu \mathrm{L})$ & $134-523(71)$ & $317 \pm 78.2$ \\
ESR $(\mathrm{mm} / \mathrm{hr})$ & $3-14(21 / 71,29.6 \%)$ & $9.6 \pm 3.4$ \\
$\quad<15$ & $15-90(50 / 71,70.4 \%)$ & $38.1 \pm 16.7$ \\
$\quad \geq 15$ & & $15.4 \pm 12.8$ \\
CRP Titer & $2.4-48(22 / 71,31 \%)$ & \\
$\quad$ Positive & $0(49 / 71,69.0 \%)$ & \\
$\quad$ Negative & & \\
Serum amyloid A (SAA) $(\mathrm{mg} / \mathrm{L})$ & $13.7-29.5(15 / 71,21.1 \%)$ & $81.62 \pm 31.6$ \\
$\quad<30 \mathrm{mg} / \mathrm{L}$ & $32.6-129.2(56 / 71,78.9 \%)$ & 4.8 \\
$\quad \geq 30 \mathrm{mg} / \mathrm{L}$ &
\end{tabular}

Number in parentheses is number of patients.

Only 5 patients (7\%) had TLC $13.7-23.4 \times 10^{3}$ per microliter of blood.

The median SAA level was $76.1 \mathrm{mg} / \mathrm{L}$ (for the range $13.7-129.2 \mathrm{mg} / \mathrm{L}$ ).

between SAA level and either ESR (correlation coefficient -0.105 and $P$ value 0.382 ) or CRP level (correlation coefficient -0.125 , with $P$ value 0.579 ).

When stratified according to the level of serum amyloid, whether low $(<30 \mathrm{mg} / \mathrm{L})$ or high $(\geq 30 \mathrm{mg} / \mathrm{L})$, no statistically significant correlation could be found between SAA levels and demographic, clinical characteristics and type of gene mutation (Table 3).

It is worth noting that high SAA levels are more frequent in female than in male patients ( $91.4 \%$ and $66.7 \%$, resp.) and are associated with more clinical manifestations during attacks as well as increased ESR and positive CRP. Amongst patients having high SAA levels, 39 patients $(39 / 56,69.6 \%)$ showed increased ESR, and 16 patients out of 22 patients having positive CRP (72.7\%) had high SAA.

Further, higher mean SAA levels were more frequent in patients with heterozygous and compound heterozygous patients than the homozygous patients were, though the difference was statistically insignificant.

The relations of SAA level to the dose of colchicine used and duration of treatment as well as adherence to treatment are presented in Table 4.

The mean SAA levels were higher in patients on low colchicine dose compared to patients on high colchicine dose $(70.0 \pm 37.1 \mathrm{mg} / \mathrm{L}$ and $68.4 \pm 35.9 \mathrm{mg} / \mathrm{L}$, resp., with $P$ value $<$ $0.9)$, though the differences were statistically insignificant.

\section{Discussion}

The aim of the present study was to measure the SAA levels in Egyptian FMF children two weeks following the last FMF attack, as a marker of subclinical inflammatory process during these periods, correlating the SAA levels with demographic, clinical, and laboratory variables, colchicine dosage, and adherence to treatment.

Seventy-eight percent $(78.9 \%)$ of the study group had high SAA levels two weeks after the last FMF attack. The results agree with those of Duzova et al. [15] who found
SAA levels above the normal range in more than $95 \%$ of their studied children with FMF in between the attacks. On the other hand, Lachmann et al. [7] and Berkun et al. [12] reported elevated SAA in only $33.3 \%$ and $25 \%$ of their FMF patients, respectively, during attack-free periods. The discrepancy between their results and the present findings may be due to the difference in the studied age groups and perhaps to the difference in the compliance of patients to colchicine. Additional factor is the inability to give high therapeutic doses of colchicine in a considerable number of children due to intolerance and occurrence of complications, mainly diarrhea.

Further, CRP was found positive in $31 \%$ of patients in the present study which is in concordance with the findings of Duzova et al. (CRP positive in 37.7\%) [15] and Korkmaz et al. (CRP positive in 34\%) [9]. Also, Lachmann et al. [7] reported that CRP was higher than normal in FMF patients during attack-free periods compared to healthy controls. Yet, no correlation was found between SAA and CRP in the present study, unlike reports of Duzova et al. [15] and Berkun et al. [12] who found high correlation between SAA levels and CRP.

The elevated SAA levels in about $79 \%$ of patients and positive CRP in about $1 / 3$ of patients, together with increased ESR in about $2 / 3$ of patients, observed in the present study, point to continuation of the inflammatory process during the attack-free periods. Further, it appears that SAA is a better indicator of the subclinical inflammation.

Moreover, the present findings revealed that high SAA was significantly associated with the female gender. It was more commonly present in children of consanguineous parents and those with family history of FMF and renal disease. Berkun et al. [12] reported that elevated SAA was significantly associated with family history of FMF and high serum CRP but not with age or gender.

In the present work higher SAA values were encountered in patients on low colchicine dose, though the difference was statistically insignificant compared to patients on high colchicine dose. Duzova et al. [15] reported similar findings. 
TABLE 3: Demographic and clinical characteristics of studied patients stratified according to the level of SAA.

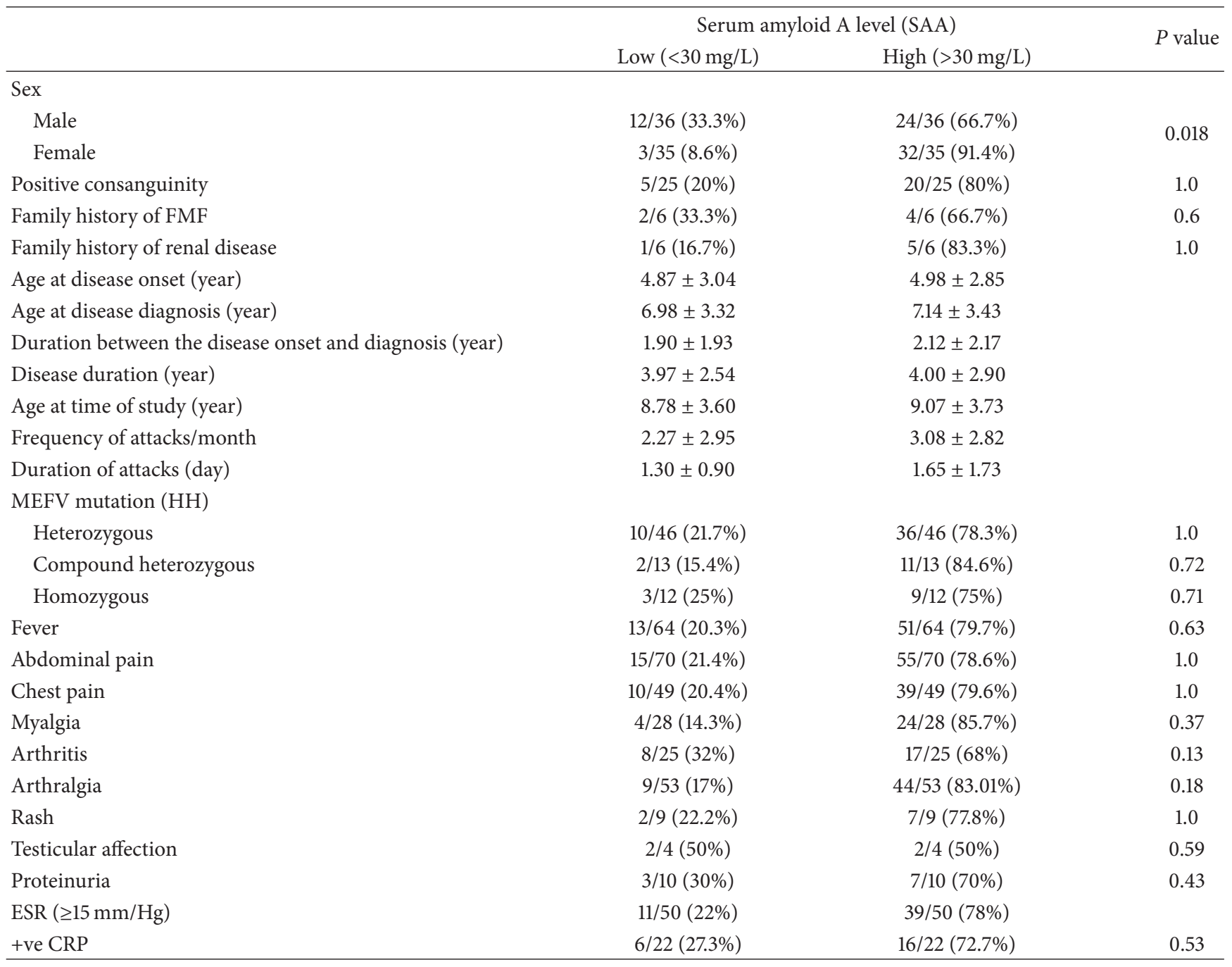

TABLE 4: Correlation between colchicine treatment and SAA level.

\begin{tabular}{lcc}
\hline Colchicine treatment & Low level $(<30 \mathrm{mg} / \mathrm{L})$ & SAA level \\
$(n=15)$ & $\begin{array}{c}\text { High level }(\geq 30 \mathrm{mg} / \mathrm{L}) \\
(n=56)\end{array}$ & $1.86 \pm 1.83$ years \\
\hline $\begin{array}{l}\text { Duration of colchicine treatment } \\
\text { Colchicine dosage }\end{array}$ & $2.0 \pm 1.56$ years & 43 patients $(81.1 \%)$ \\
Low dose $(<1.5 \mathrm{mg} /$ day) & 10 patients $(18.9 \%)$ & 13 patients $(72.2 \%)$ \\
High dose $(\geq 1.5 \mathrm{mg} /$ day $)$ & 5 patients $(27.8 \%)$ & 32 patients $(82 \%)$ \\
Adherence to colchicine & 7 patients $(18 \%)$ & \\
\hline
\end{tabular}

Note: $(n)=$ number of patients.

These findings indicate that nonresponse to colchicine may be partly due to incompliance due to complications or socioeconomic factors. Effort should be done to improve compliance by minimizing the complications and ensuring regular supply of the medication through health insurance system. The difference may be also attributed to the effect of individual factors on the serum level of the drug compared to the level of colchicine within the granulocytes, determined by p-glycoprotein pump [21]. Moreover, the measurement of SAA can be used to detect patients with poor compliance, which is not always perfect in children with FMF. Different forms of incompliance were reported in our study group; as some patients may stop therapy on their own, others may use less than the recommended colchicine dose once the severity 
of attacks subsides or the frequency decreases. Moreover, children encounter frequent complications from colchicine mainly diarrhea, so in spite of being adherent to colchicine, full therapeutic dose cannot be reached.

It is, however, worth mentioning that following recapitulation of the present results, the dose of colchicine was increased in some patients with high SAA levels, together with a trial to increase the tolerance by dose splitting and urge patients to adhere to colchicine therapy.

High SAA levels were detected two weeks after last FMF attack in a large percentage of Egyptian FMF children. This indicates that subclinical inflammation continues during attack-free periods, and SAA could be used as a marker of it. Measurement of SAA in a larger number of patients after increasing the colchicine dose and improving the compliance may help in decreasing the risk of amyloidosis.

The high levels of SAA may be also due to incompliance in about $45 \%$ of Egyptian FMF children. Effort should be directed towards improving colchicine tolerance and providing regular supply of colchicine for FMF Egyptian children. Further studies to decrease gastrointestinal side effects of colchicine may be beneficial especially in children.

\section{Disclosure}

The work was done at Rheumatology Clinic, Pediatric Specialized Hospital, Cairo University, and it was funded by the authors.

\section{Competing Interests}

The authors declare that there is no conflict of interests regarding the publication of this paper.

\section{Authors' Contributions}

Hala M. Lofty conducted revision of statistical analysis of the results and revision of the manuscript. Huda Marzouk contributed idea, data collection, revision of the statistical analysis of the result, writing and revising the results, and writing the manuscript. Yomna Farag performed data collection, revision of the statistical analysis of the results, and revision of manuscript. Mohammad Nabih conducted supervision of data collection and revision of the manuscript. Iman A. S. Khalifa performed data collection and revision of manuscript, being the corresponding author for publication. Noha Mostafa performed data collection and revision of manuscript. Ahmed Salah conducted revision of results and statistical analysis. Laila Rashed contributed performance and supervision of laboratory investigations and revision of manuscript. Kamal El Garf conducted revision of the statistical analysis of the results and revision of manuscript.

\section{References}

[1] E. Ben-Chetrit and I. Touitou, "Familial mediterranean fever in the world," Arthritis Care and Research, vol. 61, no. 10, pp. 14471453, 2009.
[2] C. Fonnesu, C. Cerquaglia, M. Giovinale et al., "Familial mediterranean fever: a review for clinical management," Joint Bone Spine, vol. 76, no. 3, pp. 227-233, 2009.

[3] French FMF Consortium, "A candidate gene for familial Mediterranean fever," Nature Genetics, vol. 17, no. 1, pp. 25-31, 1997.

[4] M. Lidar and A. Livneh, "Familial mediterranean fever: clinical, molecular and management advancements," Netherlands Journal of Medicine, vol. 65, no. 9, pp. 318-324, 2007.

[5] A. Livneh, P. Langevitz, D. Zemer et al., "Criteria for the diagnosis of familial Mediterranean fever," Arthritis and Rheumatism, vol. 40, no. 10, pp. 1879-1885, 1997.

[6] Y. Berkun and E. M. Eisenstein, "Diagnostic criteria of familial Mediterranean fever," Autoimmunity Reviews, vol. 13, no. 4-5, pp. 388-390, 2014.

[7] H. J. Lachmann, B. Şengül, T. U. Yavuzşen et al., "Clinical and subclinical inflammation in patients with familial Mediterranean fever and in heterozygous carriers of MEFV mutations," Rheumatology, vol. 45, no. 6, pp. 746-750, 2006.

[8] M. Tunca, G. Kirkali, M. de Soytürk, S. Akar, M. B. Pepys, and P. N. Hawkins, "Acute phase response and evolution of familial Mediterranean fever," The Lancet, vol. 353, no. 9162, p. 1415, 1999.

[9] C. Korkmaz, H. Özdogan, O. Kasapçopur, and H. Yazici, "Acute phase response in familial mediterranean fever," Annals of the Rheumatic Diseases, vol. 61, no. 1, pp. 79-81, 2002.

[10] Y. Bilginer, T. Akpolat, and S. Ozen, "Renal amyloidosis in children," Pediatric Nephrology, vol. 26, no. 8, pp. 1215-1227, 2011.

[11] G. Merlini and V. Bellotti, "Molecular mechanisms of amyloidosis," New England Journal of Medicine, vol. 349, no. 6, pp. 583596, 2003.

[12] Y. Berkun, S. Padeh, B. Reichman et al., "A single testing of serum amyloid A level as a tool for diagnosis and treatment dilemmas in familial Mediterranean fever," Seminars in Arthritis and Rheumatism, vol. 37, no. 3, pp. 182-188, 2007.

[13] T. Kallinich, D. Haffner, T. Niehues et al., "Colchicine use in children and adolescents with familial Mediterranean fever: literature review and consensus statement," Pediatrics, vol. 119, no. 2, pp. e474-e483, 2007.

[14] S. Özen, Y. Bilginer, N. A. Ayaz, and M. Calguneri, "Anti-interleukin 1 treatment for patients with familial mediterranean fever resistant to colchicine," Journal of Rheumatology, vol. 38, no. 3, pp. 516-518, 2011.

[15] A. Duzova, A. Bakkaloglu, N. Besbas et al., "Role of A-SAA in monitoring subclinical inflammation and in colchicine dosage in familial Mediterranean fever," Clinical and Experimental Rheumatology, vol. 21, no. 4, pp. 509-514, 2003.

[16] E. Ben-Chetrit and S. Aamar, "About colchicine compliance, resistance and virulence," Clinical and Experimental Rheumatology, vol. 27, supplement 53, no. 2, pp. S1-S3, 2009.

[17] F. Yalçinkaya, S. Özen, Z. B. Özçakar et al., "A new set of criteria for the diagnosis of familial mediterranean fever in childhood," Rheumatology, vol. 48, no. 4, pp. 395-398, 2009.

[18] C. R. Kind and M. B. Pepys, "The role of C-reactive protein (CRP) measurement in clinical practice," Journal of Internal Medicine, vol. 5, pp. 112-151, 1984.

[19] A. Biopong and J. Burthem, "Supplementary techniques including blood parasite," in Practical Hematology, B. J. Bain, I. Bates, M. A. Laffan, and S. M. Lewis, Eds., vol. 6, p. 102, Elseiver, 2012.

[20] T. Nakayama, S. Sonoda, T. Urano, T. Yamada, and M. Okada, "Monitoring both serum amyloid protein $\mathrm{A}$ and C-reactive 
protein as inflammatory markers in infectious diseases," Clinical Chemistry, vol. 39, no. 2, pp. 293-297, 1993.

[21] W. T. Klimecki, B. W. Futscher, T. M. Grogan, and W. S. Dalton, "P-glycoprotein expression and function in circulating blood cells from normal volunteers," Blood, vol. 83, no. 9, pp. 24512458, 1994. 


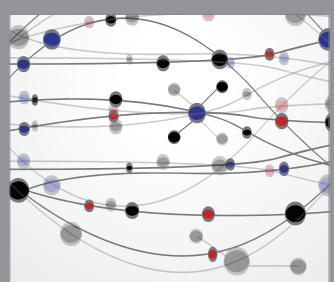

The Scientific World Journal
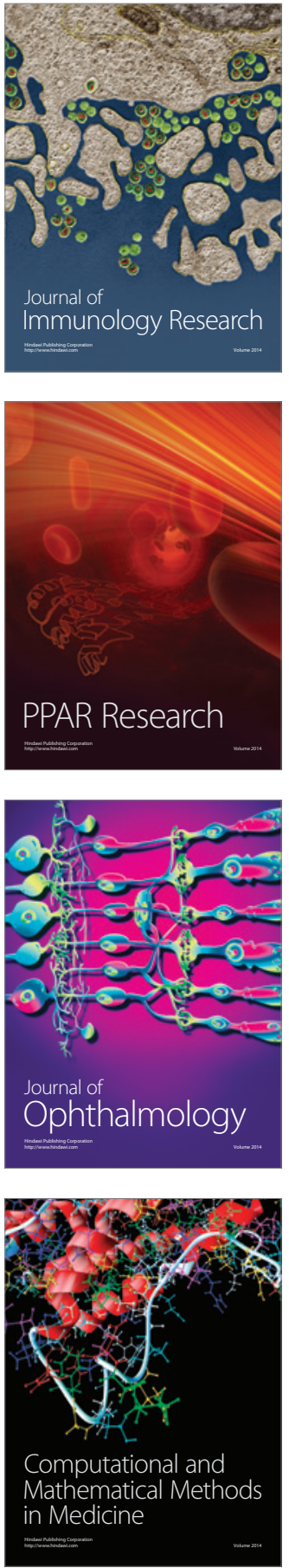

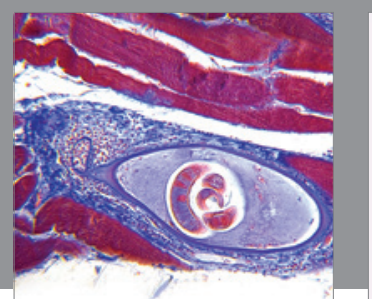

Gastroenterology Research and Practice

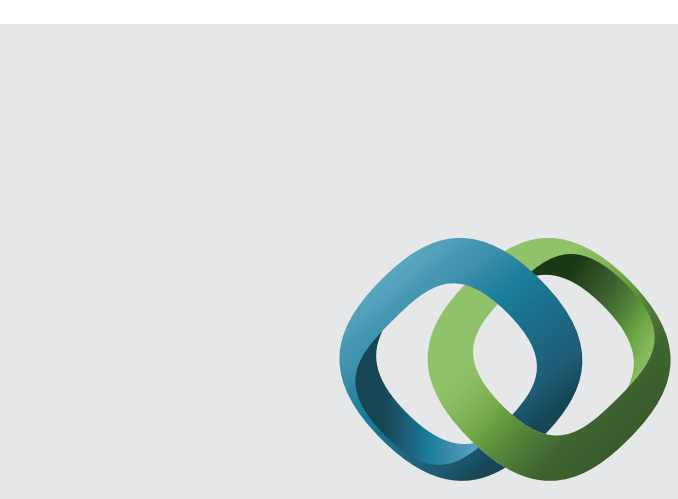

\section{Hindawi}

Submit your manuscripts at

http://www.hindawi.com
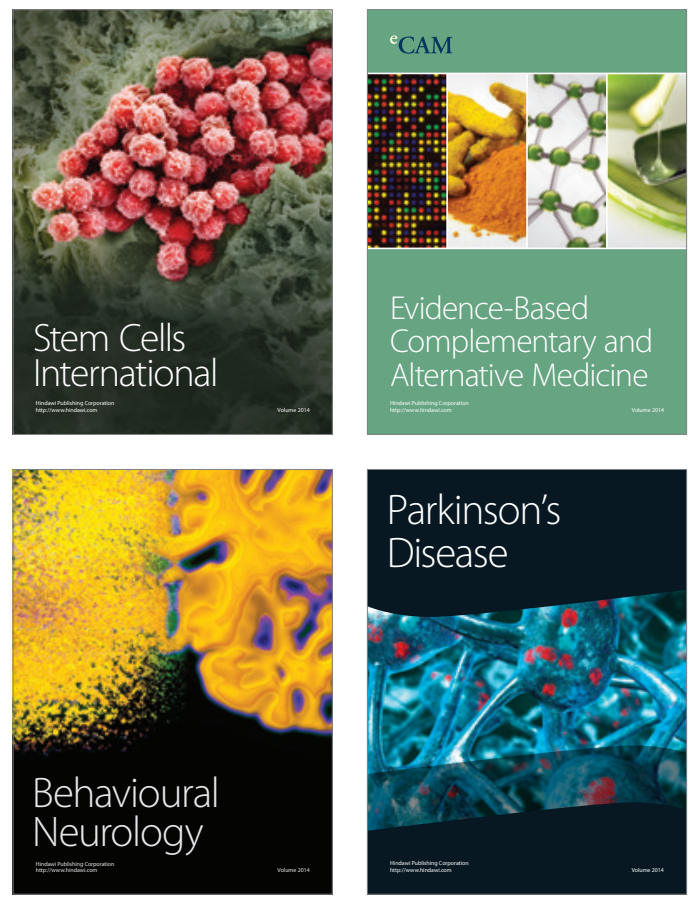
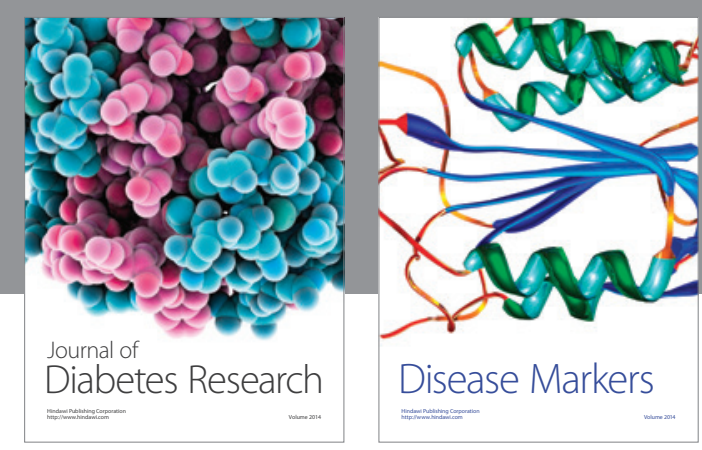

Disease Markers
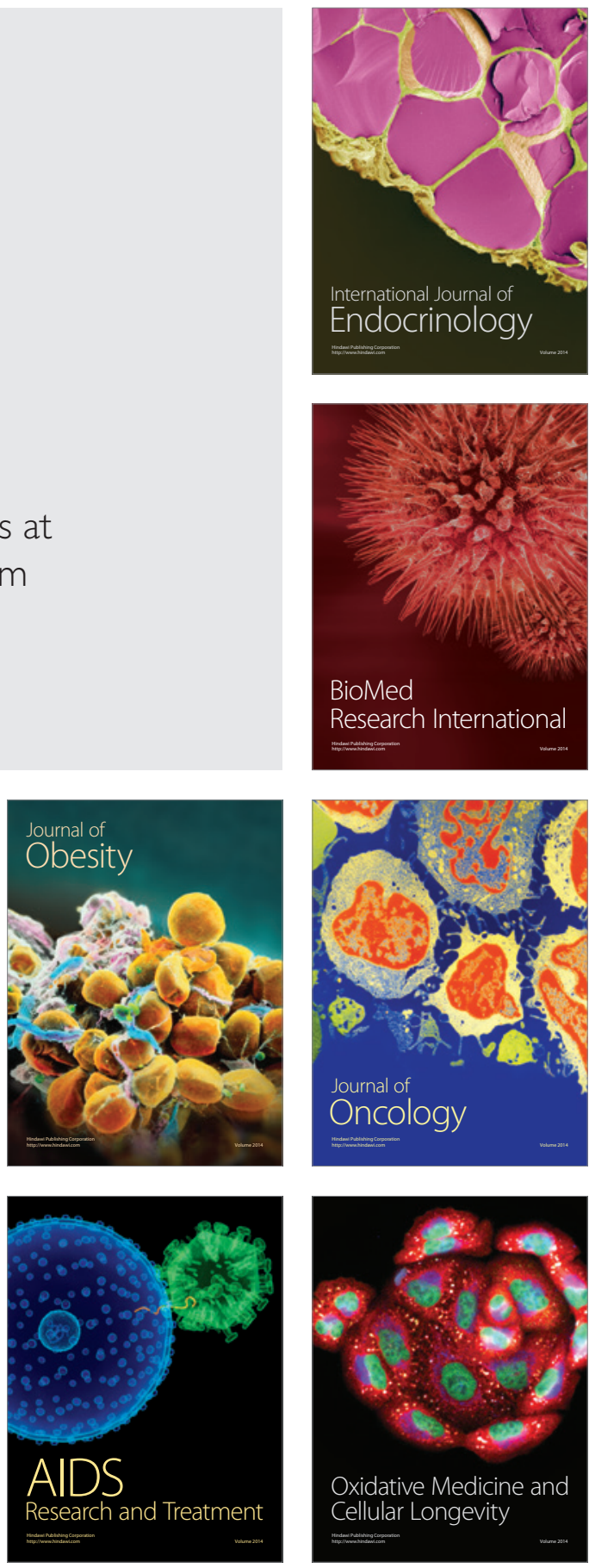